\title{
Influence of single physical loads of different intensity on the secretory activity of athletes blood neutrophils and monocytes
}

\author{
Elena Kapustina ${ }^{1}$, Olesya Ivanova $^{2}$, Sergey Usenko $^{1}$, Ludmila Demyanova $^{3}$, and Irina \\ Usova $^{4, *}$ \\ ${ }^{1}$ Southern Federal University, Bolshaya Sadovay St. 105, Rostov-on-Don, Rostov region, 344000, \\ Russia \\ ${ }^{2}$ Moscow State University of Technologies and Management named after K.G. Razumovskiy (The \\ First Cossack University), Zemlyanoy Val St. 73, Moscow, Russia \\ ${ }^{3}$ Don State Technical University, Gagarin Square 1, Rostov-on-Don, Rostov region, 344003, Russia \\ ${ }^{4}$ Institute of Technologies (branch) of DSTU in Volgodonsk, Mira Avenue 16, Volgodonsk, Rostov \\ region, 347386, Russia
}

\begin{abstract}
The article studies the impact of one-time physical activity of varying intensity on the secretory activity of neutrophils and monocytes in the blood of athletes of different sports specialization. Found that a single physical load average and peak levels experienced by athletes involved in aerobics, football and ran middle distance, cause a similar decrease in the expression of the secretory activity of neutrophils and monocytes in the blood, which is a decrease in the secretion of IL-1 $\beta$, IL- 6 , IL- 8 , TNF- $\alpha$ and $\alpha$ IFN. Single physical loads threshold adverse effect on the functional activity of neutrophils and monocytes in athletes of different specialization do not have.
\end{abstract}

\section{Introduction}

The secretory function of neutrophils and monocytes is one of the most important functions in the implementation of intercellular communication of immunocompetent cells [1-2]. Disruption of the secretory activity of monocytes and neutrophils under the influence of prolonged, systematic physical exertion occurring during the training macrocycle was previously noted in athletes of different sports qualifications [3-6]. It was found that longterm, systematic physical activity of varying intensity has a different in severity inhibitory effect on the secretory function of neutrophils and peripheral blood monocytes [7-9]. The greatest suppression of the secretion of cytokines in monocytes and neutrophils is caused by prolonged, systematic physical activity of the peak level, moderate suppression - by physical activity of medium intensity, the least negative effect is exerted by physical activity at the threshold level.

\footnotetext{
* Corresponding author : irina_usova@mail.ru
} 
The secretory activity of monocytes and neutrophils also significantly differs, depending on the period of the training macrocycle [10-11]. The greatest negative influence of physical activity on the secretory function of neutrophils and monocytes is observed in the competitive and preparatory periods under the influence of physical activity of the peak level, the least in the transition period under the influence of physical activity at the threshold level.

The influence of single physical loads of different intensity on the secretory activity of neutrophils and blood monocytes of athletes of different sports specialization has not been previously studied.

The aim of this study was to study the secretory activity of neutrophils and peripheral blood monocytes in athletes of different sports specialization under the influence of physical loads at the threshold, average and peak levels during single training sessions.

\section{Research methods and organization}

There were 129 athletes under observation, 43 persons of them were engaged in aerobics, 47 were engaged in football, 39 were engaged in middle distance running. Among them there were 81 men $(62.8 \%)$ and- $48(37.2 \%)$ women. Among 43 athletes involved in aerobics, there were 12 men $(27.9 \%)$, women - $31(72.1 \%)$. among39 athletes involved in middle distance running, there were 17 women (43.6\%), and 22 men (56.4\%). All athletes involved in football were male. The age of the observed athletes was 18-25 years old (average age $21.7 \pm 1.1$ years).

The experience of playing sports in 8 athletes $(6.2 \%)$ was less than 2 years, in 49 athletes $(37.9 \%)$ from 2.1 to 3 years, in 65 athletes (50.4\%) from 3.1 to 4 years, 7 athletes $(5.4 \%)$ have more than 5 years. In terms of sports qualifications, the observed athletes were distributed as follows: athletes of mass categories - 107 people $(82.9 \%)$, candidates for master of sports -13 people $(10.1 \%)$, master of sports -9 people $(7.0 \%)$.

The group of the reference norm consisted of 45 people aged 18-25 years (28 men $(62.2 \%)$ and 17 women $(37.8 \%)$ ) who did not regularly go in for sports and were practically healthy at the time of the study.

The level of physical activity experienced by athletes during the training cycle was judged on the basis of the maximum oxygen consumption (MOC). The determination of the maximum oxygen consumption was carried out according to the method of Astard P.O., Ryhming J.A. [12], calculations were performed using a nomogram. The level of physical activity was calculated using the formula:

$$
\mathrm{W}=\mathrm{BW} \times \mathrm{H} \times \mathrm{T} \times 1.33
$$

where $\mathrm{W}$ is the load $(\mathrm{kg} \times \mathrm{m} / \mathrm{min})$; $\mathrm{BW}$ - body weight $(\mathrm{kg})$; $\mathrm{H}-$ step height $(\mathrm{m})$; $\mathrm{T}$ is the number of ascents in 1 min., 1.33 is the coefficient of costs for descent.

Blood for research was taken from a vein. Neutrophils were isolated on a double ( $p=$ 1.095) density gradient of ficoll-verographin according to the modified method of Kheifets L.B. (1973) [13]. Monocytes from peripheral blood were isolated by the method of Mikheenko T.V. (1987) [14]. The purity of the monocyte suspension (89-98\%) was confirmed by the immunofluorescence method using monoclonal antibodies to CD14 receptors. The viability of cells in the suspension was confirmed in the trypan blue test (it was $89-93 \%$ ).

Determination of interleukins (IL-1 $\beta$, IL-2, IL-4, IL-6, IL-8), tumor necrosis factor $\alpha$ $(\mathrm{TNF} \alpha)$ and interferon- $\alpha$ (IFN $\alpha)$ in supernatants of cultures of neutrophils and blood monocytes of athletes was carried out by enzyme immunoassay on an automatic immunoassay complex «GBG Star Fax 2100» manufactured by «Awareness Technology 
Inc.» (USA) using commercial test systems manufactured by Gen-Probe Diaclone (France), in accordance with the instructions on the study procedure for each of the above mediators.

All studies were carried out twice: before training and after training with physical activity of the threshold, average and peak levels, separately in athletes involved in aerobics, football, and middle distance running.

Statistical processing of the data obtained was carried out using the student's test [15].

Results of the research and their discussion: It was found that under the influence of physical exertion, experienced by athletes of different specializations, the inhibition of the secretory activity of neutrophils and blood monocytes occurs.

At the same time, the severity of the decrease in the secretory function of these populations of leukocytes depends on the level of physical activity, and, to a lesser extent, on the kind of sport that athletes are engaged in. The negative effect of physical activity on the secretory ability of neutrophils and blood monocytes of athletes is expressed in a decrease in the unstimulated secretion of a number of mediators, such as IL-1 $\beta$, IL- 6 , IL-8, TNF- $\alpha$, IFN- $\alpha$.

As follows from Table 1, single physical loads of the threshold level did not significantly affect the secretory function of neutrophils and peripheral blood monocytes in athletes. This was evidenced by the registered levels of the studied mediators, which, after training in the indicated load mode, did not have statistically significant differences with similar indicators of the reference norm. There was also no significant difference between the levels of secretion of the studied mediators before and after training.

Table 1. Secretory activity of blood neutrophils and blood monocytes in the general group of athletes, depending on the level of physical activity during training $(\mathrm{M} \pm \mathrm{m})$

\begin{tabular}{|c|c|c|c|}
\hline $\begin{array}{c}\text { Indicator } \\
\mathrm{pg} / \mathrm{ml}\end{array}$ & Reference norm $(n=45)$ & Before training ( $n=129)$ & After training $(\mathrm{n}=129)$ \\
\hline \multicolumn{4}{|c|}{ Physical activity threshold level } \\
\hline \multicolumn{4}{|c|}{ Neutrophils } \\
\hline IL-1 $\beta$ & $3.4 \pm 0.15$ & $3.3 \pm 0.17$ & $3.3 \pm 0.16$ \\
\hline IL-6 & $1.6 \pm 0.1$ & $1.6 \pm 0.08$ & $1.4 \pm 0.07$ \\
\hline IL-8 & $4.5 \pm 0.2$ & $4.5 \pm 0.02$ & $4.1 \pm 0.2$ \\
\hline TNF- $\alpha$ & $8.7 \pm 0.3$ & $8.8 \pm 0.4$ & $8.2 \pm 0.4$ \\
\hline \multicolumn{4}{|c|}{ Monocytes } \\
\hline IL-1 $\beta$ & $17.3 \pm 0,9$ & $17.5 \pm 0.9$ & $15.3 \pm 0.8$ \\
\hline IL-6 & $4.2 \pm 0.2$ & $4.1 \pm 0.2$ & $3.7 \pm 0.2$ \\
\hline IL-8 & $2.1 \pm 0.1$ & $2.1 \pm 0.1$ & $1,9 \pm 0.1$ \\
\hline TNF- $\alpha$ & $7.1 \pm 0.3$ & $7.2 \pm 0.35$ & $6.4 \pm 0.3$ \\
\hline IFN- $\alpha$ & $11.3 \pm 0.5$ & $11.3 \pm 0.6$ & $10.7 \pm 0.5$ \\
\hline \multicolumn{4}{|c|}{ Medium physical activity } \\
\hline \multicolumn{4}{|c|}{ Neutrophils } \\
\hline IL-1 $\beta$ & $3.4 \pm 0.15$ & $3.5 \pm 0.17$ & $2.8 \pm 0.14 * *$ \\
\hline IL-6 & $1.6 \pm 0.1$ & $1.6 \pm 0.08$ & $1.25 \pm 0.06^{* *}$ \\
\hline IL-8 & $4.5 \pm 0.2$ & $4.6 \pm 0.2$ & $3.7 \pm 0.2 * *$ \\
\hline TNF- $\alpha$ & $8.7 \pm 0.3$ & $8.6 \pm 0.4$ & $7.4 \pm 0.35 * *$ \\
\hline \multicolumn{4}{|c|}{ Monocytes } \\
\hline IL-1 $\beta$ & $17.3 \pm 0.9$ & $17.2 \pm 0.9$ & $14.5 \pm 0.7^{*}$ \\
\hline IL-6 & $4.2 \pm 0.2$ & $4.3 \pm 0.2$ & $3.5 \pm 0.17 *$ \\
\hline IL-8 & $2.1 \pm 0.1$ & $2.1 \pm 0.1$ & $1.7 \pm 0.09 * *$ \\
\hline TNF- $\alpha$ & $7.1 \pm 0.3$ & $7.1 \pm 0.35$ & $6.0 \pm 0.3 *$ \\
\hline IFN- $\alpha$ & $11.3 \pm 0.5$ & $11.4 \pm 0.6$ & $9.8 \pm 0.5^{*}$ \\
\hline \multicolumn{4}{|c|}{ Peak physical activity } \\
\hline
\end{tabular}




\begin{tabular}{|l|c|c|c|}
\hline \multicolumn{5}{|c|}{ Neutrophils } \\
\hline IL-1 $\beta$ & $3.4 \pm 0.15$ & $3.3 \pm 0.16$ & $2.2 \pm 0.1^{* * *}$ \\
\hline IL-6 & $1.6 \pm 0.1$ & $1.6 \pm 0.08$ & $0.9 \pm 0.05^{* * *}$ \\
\hline IL-8 & $4.5 \pm 0.2$ & $4.4 \pm 0.2$ & $2.9 \pm 0.15^{* * *}$ \\
\hline TNF- $\alpha$ & $8.7 \pm 0.3$ & $8.6 \pm 0.4$ & $6.0 \pm 0.3^{* * *}$ \\
\hline \multicolumn{4}{|c|}{ Monocytes } \\
\hline IL-1 $\beta$ & $17.3 \pm 0.9$ & $17.2 \pm 0.9$ & $11.9 \pm 0.6^{* * *}$ \\
\hline IL-6 & $4.2 \pm 0.2$ & $4.1 \pm 0.2$ & $1.3 \pm 0.06^{* * *}$ \\
\hline IL-8 & $2.1 \pm 0.1$ & $2.1 \pm 0.1$ & $4.7 \pm 0.2^{* * *}$ \\
\hline TNF- $\alpha$ & $7.1 \pm 0.3$ & $7.1 \pm 0.35$ & $8.7 \pm 0.4^{* * *}$ \\
\hline IFN- $\alpha$ & $11.3 \pm 0.5$ & $11.2 \pm 0.6$ & \\
\hline
\end{tabular}

Note: $*$ - $p<0.05, * *-p<0.01, * * *-p<0.001$ compared to the reference norm.

Conducting trainings among athletes of the general group in the mode of single physical loads of an average level caused a significant decrease in the secretory activity of both neutrophils and monocytes. The initial level of secretion of mediators by monocytes and peripheral blood neutrophils before training did not differ from those for the reference norm.

After a single physical activity of an average level, the secretion of IL-1 $\beta$ by neutrophils was 1.21 times lower than the reference norm, IL- $6-1.28$ times, IL- $8-1.22$ times, and TNF- $\alpha-1.18$ times lower $(\mathrm{p}<0.01)$. In the monocyte population, the secretion of IL- $1 \beta$ was reduced relative to the reference norm by 1.19 times, IL- 6 - by 1.2 times, IL- 8 - by 1.24 times, TNF- $\alpha$ - by 1.18 times, and IFN- $\alpha$ - by 1,15 times $(\mathrm{p}<0.05 \mathrm{p}<0.01)$.

Under the influence of single physical exertion of the peak level, the secretion of IL-1 $\beta$ by neutrophils was 1.55 times lower than the reference norm, IL-6 - 1.78 times lower, and the secretion of IL-8 and TNF $\alpha-1.55$ and 1.45 times, respectively (in all comparisons $p$ $<0.001)$. In cultures of monocytes it was 1.45 times lower than the reference norm, the secretion of IL-6 was lower by 1.44 times, IL8 was lower by 1.62 times, and TNF- $\alpha$ and IFN was lower by 1.51 and 1.3 times., respectively $(\mathrm{p}<0.001$ in all the above comparisons.

Single physical loads of average and peak levels experienced by athletes involved in aerobics, football and middle-distance running cause a similar decrease in the secretory activity of neutrophils and blood monocytes, which is manifested in a decrease in the secretion of IL-1 $\beta$, IL- 6 , IL-8 and TNF- $\alpha$. Single physical loads of a threshold level do not have a negative effect on the functional activity of neutrophils and monocytes in athletes of different specializations. It is proposed to exclude from the training cycle athletes involved in aerobics, football and middle-distance running, physical activity of the peak level or significantly reduce the number of such trainings in order to prevent the occurrence of an immunodeficiency state and metabolic disorders. Research prospects are in the further study of immune and metabolic parameters, which will optimize the training process and develop criteria for screening the immune and metabolic status of athletes.

\section{Conclusion}

One-time training with the intensity of physical activity of medium and peak levels, experienced by athletes engaged in aerobics, football and middle distance running, cause the same manifestation of changes in acid resistance of erythrocytes of peripheral blood of athletes, which is manifested in reduced erythrocyte spherulation time. Maximum hemolysis, as well as in increasing the duration of hemolysis, height and number of hemolysis maxima.

Exercise of the threshold level during one-time training does not have a negative impact on the acid resistance of erythrocytes in athletes of different sports specialization. 
One-time training with the intensity of physical activity of medium and peak levels, experienced by athletes engaged in aerobics, football and middle distance running, cause in peripheral blood erythrocytes the same degree of manifestation.

Exercise of the threshold level during one-time training does not cause a negative effect on the sex and the system of erythrocytes in athletes of different sports specialization.

One-time training with the intensity of physical activity of medium and peak levels, experienced by athletes engaged in aerobics, football and middle distance running, cause the same manifestations of changes in the system of adenyl nucleotides, which is manifested in a decrease in intracellular ATP concentration, increase intracellular ADP and AMP, and in the formation of a tendency to decrease the erythrocytes.

Threshold-level physical activity during one-time training does not have a negative effect on the system of adenyl nucleotides of erythrocytes in athletes of different sports specialization.

One-time training with the intensity of physical activity of medium and peak levels, experienced by athletes engaged in aerobics, football and middle distance running, cause the same manifestation of changes in phagocytic and secretory activity of neutrophils and blood monocytes of athletes, which is manifested in reduced PF and FI, as well as in the suppression of secretion of IL-1 $\beta$, IL- 6 , IL-8, TNF- $\alpha$ and IFN- $\alpha$. Exercise of the threshold level during one-time training does not cause a negative impact on the functional activity of neutrophils and monocytes in athletes of different sports specializations.

One-time training with the intensity of physical activity of medium and peak levels, experienced by athletes engaged in aerobics, football and middle distance running, cause significant changes in the population composition of lymphocytes, which is manifested in a decrease in $\mathrm{CD} 3+, \mathrm{CD} 4+, \mathrm{CD} 16+$ and $\mathrm{CD} 22+$ lymphocytes with tendency to decrease the immunoregulatory index CD4 / CD8, as well as decreased secretion of IL-2, IL-6, IL10 , TNF- $\alpha$ and IFN- $\gamma$. The degree of violation of the immune status of athletes, regardless of their sports specialization, is similar at the same level of physical activity. The largest shifts in immune status cause one-time training during peak exercise.

Exercise threshold level during one-time training does not cause a negative impact on the number and population composition of blood lymphocytes in athletes of different sports specializations.

\section{References}

1. C. Rambarat, F. Reifsteck, Y. Taha, J. Clugston, Journal of the American College of Cardiology, 75, 14 (2020).

2. M. Azizi, Procedia-Social and Behavioral Sciences, 15, 1556-1560 (2011)

3. J. Kropa, J. Close, D. Shipon, The Journal of Pediatrics, 178, 194-199 (2016)

4. I. Jonsdottir , P. Schjerling, K. Ostrowski, Journal of Physiology (London), 528, 157$163(2000)$

5. A.-S. Schreurs, S. Torres, T. Truong, E. L. Moyer, A. Kumar, C. G. T. Tahimic, J. S. Alwood, American Journal of Physiology, 319, 734-C745 (2020)

6. B. K. Pedersen, L. Hoffman-Goetz, Physiological Reviews, 80, 1055-1081 (2011)

7. A. Steensberg, C. Keller, R. L. Starkie, T. Osada, M. A. Febbraio, B. K. Pedersen, American Journal of Physiology, 283, 1272-1278 (2012)

8. A. Steensberg, C. P. Fischer, M. Sacchetti, C. Keller, T. Osada, Journal of Physiology (London), 548, 631-638 (2013)

9. P. Debien, T. Timoteo, M.B. Filho, B. Miloski, C. Ferezin, Science of Gymnastics Journal, 1, 23-36 (2019) 
10. M. Penkowa, C. Keller, P. Keller, S. Jauffred , B.K. Pedersen, FASEB Journal, 17, 2166-2168 (2013)

11. P. Wicker, European Journal Management Quarterly, 3, 385-402 (2020)

12. L. Wang, J. Wang, J. Xiao, D. Cretoiu, G. Li, Journal of Sport and Health Science, 9(3), 203-210 (2020)

13. B. Williams, J. Frantz, K. Bell, M. Wainman, S. Purkayastha, S. Lyng, T. Sabo, Archives of Physical Medicine and Rehabilitation, 99, 167 (2018)

14. M. Kianmehr, M. Reza, M. Shahri,, M. Afsharnia,, Z. Rohani,, M. Ghorbani, Mutation Research, Genetic Toxicology and Environmental Mutagenesis, 837, 29-33 (2019)

15. B. R.Scott, J. P. Loenneke, K. M.Slattery, B. J. Dascombe, Journal of Sciences and Medicine in Sport, 19, 360-367 (2016) 